Didáctica Geográfica n 22, 2021, pp. 97-121

DOI: https://doi.org/10.21138/DG.577

ISSN electrónico: 2174-6451

\title{
INFANCIA Y AGENDA 2030: APRENDIENDO A PERCIBIR LA CIUDAD DESDE LAS EMOCIONES DE LOS ESCOLARES ${ }^{1}$
}

Childhood and 2030 Agenda: learning to Perceive the City from the EMOTIONS OF SCHOOL CHILDREN

ENFANCE ET AGENDA 2030: APPRENDRE À PERCEVOIR LA VILLE À PARTIR DES ÉMOTIONS DES ÉCOLIERS

\author{
Alicia González García ${ }^{\circledR}$ \\ CEIP Sebastian Elcano. Grau de Castelló. \\ alicia.elcano@gmail.com
}

Antonio José Morales Hernández

Departamento de Didáctica de las Ciencias Experimentales y Sociales (Universitat de València).

Antonio.j.morales@uv.es

Carlos Caurín Alonso

Departamento de Didáctica de las Ciencias Experimentales y Sociales (Universitat de València).

Carlos.caurin@uv.es

Recibido: 24/10/2020

Aceptado: 23/03/2021

1 Este artículo se inscribe dentro del proyecto: "Las representaciones sociales de los contenidos escolares en el desarrollo de las competencias docentes", Proyectos I+D de Generación de Conocimiento y Fortalecimiento Científico y Tecnológico del Sistema I+D+I (Ministerio de Ciencia, Innovación y Universidades), con referencia PGC2018-094491-B-C32, cofinanciado con fondos de la UE. 


\section{RESUMEN:}

El ODS 11 de la Agenda 2030 plantea la necesidad de construir y gestionar ciudades y comunidades sostenibles. En este cometido la escuela tiene un rol fundamental al evidenciar la percepción que tiene la infancia de la ciudad dónde vive desde la capacidad de despertar emociones que le puedan provocar bienestar personal y social. Esta investigación recoge un estudio de casos, realizado con alumnado de 3er curso de Educación de Primaria, que nos ha permitido relacionar representaciones sociales y emociones de los estudiantes antes y después de una salida al entorno urbano. La metodología utilizada ha sido el análisis de los dibujos realizados por los escolares de su ciudad. Mediante un diseño cuasiexperimental se ha planteado un análisis comparativo entre un grupo control que no ha realizado la salida de campo y un grupo experimental que sí. A partir de ello se evidencian unos enfoques naturalistas, antropocéntricos o globalizantes asociados a visiones idealizadas o problematizadas de la ciudad que generan emociones positivas o negativas. Los principales resultados muestran un cambio mayoritario hacia una representación globalizante desde un enfoque problematizado y emociones positivas en el grupo experimental tras la intervención. Y con ello una percepción crítica de la ciudad desde el espacio vivido al superar enfoques naturalistas y antropocéntricos a partir de visiones idealizadas mostrados por el grupo control desde la desvinculación emocional con su propio barrio.

\section{Palabras clave:}

Educación emocional; Agenda 2030; percepción; ciudad; escolares.

\section{Abstract:}

SDG11 of the 2030 Agenda raises the need to build and manage sustainable cities and communities. In this goal the school has a fundamental role in highlighting the perception of children in the city where they live from the ability to arouse emotions that can cause personal and social well-being. This research is based on a case study, conducted with students from $3^{\text {rd }}$ grade in Primary Education, has allowed us to relate social representations and emotions of students before and after an outing into the urban environment. The main results of this research show evidence of the city lived by the students from acritical citizenry. The methodology used has been the analysis of drawings made by schoolchildren in his city. By means of a quasi-experimental design, a comparative analysis has been proposed between a control group that has not made the exit through their neighborhood in their city and an experimental group that has done it. After the analysis, naturalistic, 
anthropocentric or globalizing approaches are shown associated with idealized or problematized visions of the city that generate positive or negative emotions. The main results show a majority change towards a globalizing representation from a problematized approach and positive emotions in the experimental group, after the intervention. They show a critical perception of the city based on the lived space by overcoming naturalistic and anthropocentric approaches from idealized visions shown by the control group from emotional disengagement with their own neighborhood.

\section{KeYWORDS:}

Emotional education; 2030 Agenda; perception; city; school children.

\section{RÉSUMÉ:}

L'ODD 11 de l'Agenda 2030 soulève la nécessité de construire et de gérer des villes et des communautés durables. Dans ce cadre, l'école a un rôle fondamental à jouer pour mettre en évidence la perception des enfants sur leur ville où il vit et dans sa capacité à susciter des émotions qui peuvent jouer sur leur bien-être personnel et social. Cette recherche comprend une étude de cas, menée avec des élèves de 3e année de primaire, qui a permis d'établir le lien entre les représentations sociales et les émotions des élèves avant et après une sortie dans leur environnement urbain. La méthodologie utilisée a été l'analyse des dessins réalisés par les écoliers de leur ville. Par le recours à une méthode quasi expérimentale, une analyse comparative a pu être menée entre un groupe témoin qui n'a pas effectué la sortie sur le terrain et un groupe expérimental qui l'a réalisée. L'analyse des données montre une interaction entre des approches naturalistes, anthropocentriques ou globalisantes de la ville et des visions idéalisées ou problématisées de la ville qui ont un impact sur la nature des émotions générées, qu'elles soient positives ou négatives. Les principaux résultats montrent le passage d'une vision globalisante de la ville à une représentation plus problématisée et génératrice d'émotions positives chez le groupe expérimental après l'intervention. Avec elle une perception critique de la ville et de l'espace vécu se développe, se dégageant des approches naturalistes et anthropocentriques ainsi que des visions idéalisées montrées par le groupe témoin dont le désengagement émotionnel de son propre quartier est plus marqué.

\section{Mots-CLÉs:}

Éducation émotionnelle; Agenda 2030; perception; ville; écoliers. 


\section{INTRODUCCIÓN}

La evolución disciplinar que la Geografía ha experimentado en las últimas décadas ha dejado de manifiesto que va más allá de los contenidos conceptuales; de esta manera, ha ido incluyendo las vivencias, ideología, emociones, etc. de las personas, el llamado por Capel (1973) "giro del comportamiento" supone aprender a enseñar desde el espacio vivido (Souto, 2017). Además, adentrándonos en la neurociencia, la inteligencia emocional (Salovey y Mayer, 1990; Bisquerra y Pérez Escoda, 2007; Goleman, 2010; Mora, 2018) cobra un especial protagonismo ya que contamos con evidencia de autores de que aquello que se aprende es aquello que pasa por la parte límbica de nuestro cerebro al emocionar y motivar. Para aclarar este hecho, diremos que existe un proceso que regula aprendizajes cognitivos y afectivos inhibiendo la amígdala cerebral y el hipocampo; estos núcleos forman parte del sistema límbico e intervienen en los procesos de miedo, ira y memoria también por medio de la corteza prefrontal (Gupta et al., 2018).

En este contexto, se presenta un trabajo de investigación que parte de un estudio previo inédito (González, 2019) enmarcado dentro del Máster Universitario en Investigación en Didácticas Específicas de la Universitat de València. Este estudio muestra el carácter reflexivo de una docente en activo que, desde la investigación-acción, pretende hacer un acercamiento a cómo ve y siente el alumnado de 3er curso de Educación Primaria su barrio y, si además lo hacen con una mirada crítica. El trabajo que se describe a continuación parte del análisis de las representaciones sociales que el alumnado tiene sobre su barrio, siempre en conexión con sus emociones.

La Agenda 2030 (Naciones Unidas, 2015) a través del Objetivo de Desarrollo Sostenible 11 plantea la necesidad de construir y gestionar ciudades y comunidades sostenibles. Por ello nos preguntamos cómo podemos contribuir desde la escuela a la consecución de este objetivo. Souto (2018) nos da una pista con la siguiente cita:

Sin duda el niño percibe el espacio como lo piensa y no como lo ve, tal como señala Hannoun (1977), pero lo piensa como lo siente. Y tal como han mostrado los estudios científicos de la neurociencia (Damasio, 2006, 2010; Díaz, 2009) las emociones condicionan la manera de proceder en las explicaciones. (Souto 2018, p. 15)

Esta cuestión nos lleva a plantearnos el relacionar la percepción del espacio con las emociones reflexionando cómo inciden éstas en la necesidad de construir espacios sostenibles y saludables para vivir. Es decir, es imprescindible discernir entre la ciudad percibida, construida socialmente y reproducida desde enfoques idealizados, de aquélla que vivimos y sentimos como propia. 
Todo esto justifica el objeto de esta investigación respecto a si la escuela es capaz de despertar emociones para construir ciudades y comunidades sostenibles. Por ello hemos realizado un estudio de casos con alumnado de 3er curso de Educación Primaria para indagar en las representaciones sociales que tienen sobre su ciudad y las emociones que esta les provoca utilizando como recurso didáctico la salida de campo. Todo ello con la finalidad de responder a las siguientes cuestiones: ¿siente y percibe el alumnado su ciudad de igual manera tras una salida de campo?, ¿de qué manera incide el espacio vivido en la generación de emociones?, ¿qué puede aportar todo esto al cumplimiento del ODS 11 de la Agenda 2030?

Esto nos ha permitido plantear la siguiente hipótesis: la escuela puede ayudar al cumplimento del ODS 11, utilizando como recurso didáctico la salida de campo, cuando contribuye a la conceptualización de la ciudad desde el espacio vivido y sentido por el alumnado, evidenciando con ello la problematización del entorno urbano y la necesidad de poder mejorarlo a partir de las emociones de los propios escolares. Para demostrar esta hipótesis se ha realizado un estudio de casos con 3er curso de Educación Primaria, mediante un diseño cuasiexperimental, que ha permitido el análisis comparativo del pretest y postest de un grupo experimental que ha realizado diversas salidas a la barriada próxima al centro educativo frente a otro grupo control que no ha realizado este trabajo de campo. El instrumento de investigación utilizado ha sido la representación pictórica realizada por los estudiantes sobre su ciudad, con la finalidad de analizar en sus dibujos la relación existente entre las representaciones sociales del entorno urbano y las emociones asociadas. Para ello se ha planteado un pretest y un postest.

A partir de la hipótesis, se formularon los siguientes objetivos:

1. Analizar las representaciones sociales que el alumnado tiene sobre su barrio y conectarlas con las emociones que sienten.

2. Diseñar y evaluar la aplicación de una intervención-acción en el aula abordando el análisis de las emociones del alumnado a través de las salidas de campo.

\section{MARCO TEÓRICO}

Los seres humanos somos parte del medio ambiente que nos rodea y debido a nuestros cambios evolutivos y a nuestra educación sociopolítica, podemos percibir e interpretar este medio desde diferentes puntos de vista. Nosotros interactuamos con el mismo y recibimos estímulos que interpretamos y expresamos de distintas maneras: mediante el lenguaje hablado, escrito o gestual y de forma simbólica incluyendo las representaciones sociales. Interpretando a Berger et al. (2009) y posteriormente a Hernández y Caurín (2020), podemos decir que la percepción de pertenencia al medio nos hace tomar 
conciencia de nosotros mismos promoviendo un desarrollo socioemocional positivo respecto al lugar en que vivimos.

Las distintas percepciones hacen que interpretemos la sociedad desde distintos puntos de vista e ideologías que están relacionadas con nuestra educación, nuestra genética y nuestras experiencias. Nacer en un sitio es una casualidad debida al azar, pero las razones por las que percibimos nuestra ciudad, barrio o pueblo de una manera o de otra son fruto de algunas combinaciones que desembocan en percepciones emocionales. De todo esto podemos inferir la importancia de dos vertientes en la educación: la educación emocionaly la educación por y para el medio ambiente, es decir lo que denominamos educación socioambiental (Rodríguez, 2005) que tiene muy en cuenta el cambio de actitudes como motor del cambio social (Rodríguez y Seoane, 1989). La combinación de ambos tipos de educación fomentará la formación de una ciudadanía planetaria que promueva a la naturaleza hacia un lugar privilegiado en los contextos escolares (Murga-Menoyo y Novo, 2017). Debe ser también coherente con los Objetivos de Desarrollo Sostenible (ODS) y la Agenda 2030 que promulga unas competencias clave en sostenibilidad y una justicia ambiental (Murga-Menoyo, 2018).

\subsection{Agenda 2030: la escuela y las ciudades del futuro}

La Agenda 2030 necesita de la escuela para construir las ciudades de futuro y con futuro desde su capacidad para generar emociones que favorezcan el pensamiento crítico. Por ello es imprescindible percibir la ciudad a través de la infancia para planificar y gestionar este espacio público de manera sostenible y saludable. La Convención de los Derechos del Niño (Naciones Unidas, 1989) reconoce la plena ciudadanía de la infancia. Sin embargo, estos derechos parece que se quedan sólo en teoría en lo referente a la opinión y la toma de decisiones sobre las ciudades. La ciudad se ha construido como el destino de un ciudadano varón, adulto y con trabajo, excluyendo a todos los demás y sobre todo a los niños y niñas, tercera edad y personas con diversidad funcional. En este tipo de ciudades los pequeños no pueden desarrollarse a través de actividades innovadoras y aventureras, que ofrezcan descubrimientos interesantes, que permita la superación de obstáculos adecuados y permitan un espacio compartido y un tiempo libre que fomente el juego (Tonucci, 2009).

Ciertos autores manifiestan la necesidad de estudiar la ciudad para poder transformarla (López Facal, 1987). Otros han planteado la necesidad de establecer la vinculación de la infancia con la ciudad a través del descubrimiento del medio urbano (Muntañola, 1987). Algunos han abordado, desde la didáctica, el estudio de la problemática urbana (Tomé, 1994). Y otros han puesto en evidencia el dilema existente entre enseñar la ciudad, desde el espacio percibido o aprender de la ciudad, desde el espacio vivido (Tatjer, 2001). En 
este espacio vivido es donde se llevan a cabo las relaciones interpersonales, un espacio caracterizado por las normas y la vida urbana: el colegio, el hogar, el barrio y los medios de transporte (Breda y García de la Vega, 2018).

Tonucci (2015) defiende la necesaria participación ciudadana de la infancia. Sostiene que hay que atender sus necesidades y oír sus propuestas cuando se habla de proyectos urbanísticos. Por su parte Santana, Morales y Souto (2019) manifiestan que:

Es preciso insistir en las relaciones personales para construir una participación ciudadana; o sea para pasar de la condición de persona a la de ciudadana. Pero dicha transición solo es posible desde la praxis del funcionamiento social de algunas actividades cotidianas, como son las visitas a los parques naturales. (Santana, Morales y Souto, 2019, p. 97)

A lo largo de las últimas décadas, se han realizado diversas propuestas didácticas basadas en estos principios, como el proyecto Gea-Clío, integrado por docentes de todas las etapas educativas y centrados en el desarrollo de materiales curriculares destinados a la enseñanza y aprendizaje de las Ciencias Sociales. Otro proyecto de renombre es Nos Propomos, una iniciativa de la Universidad de Lisboa en la que el alumnado de Primaria y Secundaria crea proyectos de mejora de su entorno con la finalidad de implementarlos.

Si tenemos en cuenta la Teoría de las Representaciones Sociales (Moscovici,1981; Jodelet, 1986) podemos pensar que la conceptualización de la ciudad puede quedar definida por la construcción social que se hace del propio vocablo, definida por una construcción percibida que se reproduce y transmite a través de diversos medios. Sin embargo, desde el enfoque de la Geografía Humanista y del espacio vivido es impensable aprender la ciudad sin tener en cuenta la forma en que los niños y niñas perciben emocionalmente su entorno urbano de manera significativa (Bisquerra, 2003; Bisquerra y Pérez Escoda, 2007).

Reigota (1995) identificó tres tipos de representaciones sociales relacionadas con el medio ambiente: naturalista, antropocéntrico y globalizante. Tan sólo en el último caso se evidencia una visión biocéntrica e interdisciplinar del conocimiento, imprescindible para plantear el aprendizaje desde el enfoque globalizador para desarrollar elpensamiento complejo (Zabala, 1999). Por ello es preciso partir de las propias representaciones sociales del alumnado para discernir si tienen una visión ecosistémica del entorno que les permita intuir la complejidad de relaciones que se dan en el mismo y la problematización existente. $\mathrm{Su}$ asociación emocional genera procesos de identidad (Morales, Santana y Sánchez, 2017) que les inducen a plantear propuestas para mejorar su ciudad. Y con ello 
contribuir a la consecución del ODS 11 relacionado con la construcción de ciudades y comunidades sostenibles.

\subsection{La percepción emocional del espacio}

Boira, Reques y Souto (1994) ya alertan de que el estudio de la Geografía es complejo y va más allá de factores conceptuales. En este sentido, Lefebvre (2000) ya propone una triple división del espacio: el espacio vivido, el espacio concebido y el espacio percibido. La llamada Geografía de la Percepción y del Comportamiento (o Geografía Humanista), centra el objeto de estudio en el espacio vivido.

Adentrándonos más en el estudio de las emociones, investigadores como Ekman y Oster (1981) descubrieron una serie de emociones comunes a todos los seres humanos, independientemente de su raza, cultura, sexo, etc. Formaban expresiones faciales invariables entre unos y otros y eran: la alegría, la tristeza, la ira, el desagrado y el miedo. Más adelante, en 1990, Salovey y Mayer empezaron a utilizar el término de Inteligencia Emocional y posteriormente Goleman (1995) populariza este concepto y escribe el best-seller "Emotional Intelligence". En España destacan autores como Bisquerra (2003, 2005 y 2011), López Cassà (2005), Fernández Berrocal y Extremera, (2009), Rubia (2007) que nos hablan de competencias y emociones y de la trascendencia que tienen en diferentes niveles educativos. Las emociones son producto de la sensación y de la percepción y nos ayudan a tomar conciencia propia y de lo que nos rodea; a su vez estructuran expresiones en el lenguaje que caracterizan exclusivamente a los seres humanos. Las percepciones son la interpretación que hace el cerebro de la información que recibimos a través de los sentidos y siempre está precedida por la sensación que es, según Hernández y Caurín (2020), una representación mental de la realidad. Esto ocurre en nuestro cerebro cuando detectamos estímulos que más tarde son trasformados en señales eléctricas a través de las conexiones neuronales. Estas señales se envían al cerebro a través del sistema nervioso periférico y se produce un procesamiento que nos permite apreciar el mundo que nos rodea. Después de esto, nuestro cerebro procesa esta información y da una interpretación a estas sensaciones convirtiéndolas en las percepciones que serán la base de nuestro trabajo.

Rubia (2007) nos deja claro que nuestro cerebro necesita inventar o completar la información no recibida para dar coherencia a los estímulos percibidos. Nuestro cerebro inventa una realidad que se adecúa a las leyes físicas que conocemos para dar coherencia al significado de determinado estímulo que percibimos. Por ejemplo, Rubia (2007) nos habla sobe el fenómeno "phi", que consiste en que nuestro sistema visual solo es capaz de captar imágenes fijas que se suceden una detrás de otra rápidamente (instantes en el tiempo), con breves espacios entre ellas sin información. Sin embargo, el cerebro 
completa la información entre estas imágenes fijas para que percibamos continuidad en el movimiento. Nuestro cerebro inventa parte de la realidad para que nuestra mente trabaje dentro de la lógica planteada por las leyes físicas que conocemos.

Las percepciones generan emociones que al ser razonadas nos facilitan una adaptación mayor al medio y un mayor bienestar subjetivo (Fernández Berrocal y Extremera, 2009). Si nos adaptamos mejor tenemos mayor capacidad de resolver problemas, es decir somos más competentes. Bisquerra (2003) nos habla de competencias emocionales en las que son muy relevantes el espacio y el tiempo y esa habilidad para utilizar adecuadamente el vocabulario emocional en las distintas situaciones. Si trasladamos este término al ámbito educativo, aparece el concepto de Educación Emocional que fue definida por Bisquerra (2000) de la siguiente manera:

la Educación Emocional es un proceso educativo continuo y permanente, que pretende potenciar el desarrollo emocional como complemento indispensable del desarrollo cognitivo, constituyendo ambos los elementos esenciales del desarrollo de la personalidad integral. Para ello se propone el desarrollo de conocimientos y habilidades sobre las emociones con objeto de capacitar al individuo para afrontar mejor los retos que se plantean en la vida cotidiana. Todo ello tiene como finalidad aumentar el bienestar personal y social. (Bisquerra, 2000, p. 243)

Según Abarca (2003) las emociones y su interacción social no son bidireccionales, sino que reciben la influencia de su entorno educativo. Algunas de las conclusiones emocionales realizadas por López Cassà en 2005 sobre la Educación infantil se pueden trasladar a las primeras etapas de la educación primaria como es nuestro caso, entre las que destacamos la potenciación de actitudes de respeto, tolerancia y prosocialidad. Este hecho puede estar relacionado con el concepto de transformar las emociones en acciones positivas, la llamada ecología emocional (Soler y Conangla, 2004).

\section{METODOLOGÍA}

La investigación que se presenta en este artículo es de tipo mixto y con un diseño cuasiexperimental, dónde hubieron dos grupos (uno experimental y otro control) de 3er curso de Primaria en los que se recogieron datos para el estudio. En uno de ellos se implementó una propuesta didáctica basada en el estudio emocional del barrio y las salidas de campo, con el objetivo de comparar los resultados del grupo control con los del grupo experimental a posteriori. Por tanto, nos encontramos delante de un estudio de casos, es decir, "el estudio de la particularidad y la complejidad de un caso en singular, para llegar a comprender su actividad en circunstancias importantes" (Stake, 2005, pág. 
11). El perfil de la muestra consta de un grupo experimental $\left(3^{\circ} \mathrm{A}\right)$ constituido por 18 estudiantes, y el grupo control $\left(3^{\circ} \mathrm{B}\right)$ con 16 .

Como se ha mencionado anteriormente, el estudio nace de la motivación de la investigadora al ser también la docente de los grupos de estudiantes de la muestra en explorar un problema de interés para los mismos, es decir, hablamos del concepto de investigación-acción (Elliott, 1993).

En el diseño de la experiencia, los instrumentos de recogida de datos fueron fichas adaptadas del trabajo de investigación de Sánchez (2016) y Jerez (2018) basadas en el análisis de las representaciones pictóricas, como ya apuntaba Barraza (2000) al afirmar que el dibujo es una fuente de investigación infantil muy valiosa.

En estas fichas, la primera a modo de pretest, se les pidió a ambos grupos, que dibujasen su barrio, que lo describieran y que señalasen una emoción que les provoca al pensar en él. Posteriormente, se implementó el proyecto El Grau a vista de 3er curso de Educación Primaria únicamente en el grupo experimental. En el siguiente párrafo se explica con más detenimiento el contenido de esta propuesta educativa. En cambio, en el grupo control se siguió la propuesta del libro de texto, mediante una programación didáctica carente, principalmente, de salidas de campo. Al finalizar el proyecto en el grupo experimental y el tema del libro en el grupo control, se realizó un postest a ambos grupos, donde, además del dibujo de su barrio y la elección de la emoción que les evoca, realizaron una enumeración de aquello que les gusta, de lo que les disgusta y propuestas para mejorar su entorno próximo.

La secuencia mencionada anteriormente y que fue implementada únicamente en el grupo experimental, lleva el nombre de El Grau a vista de 3er curso de Educación Primaria y consta de tres bloques, un primer bloque de trabajo en el aula donde se analiza el mapa de la zona para familiarizarse con el plano y en el que se llevó a cabo un trabajo emocional y crítico de sensibilización a partir de imágenes que muestran problemas socialmente relevantes. En el segundo bloque, se realizaron dos salidas de campo por el barrio, en las que el alumnado anotó y fotografió aquello que quería destacar: lugares con connotaciones positivas, negativas o neutras, quejas, propuestas de mejora, etc. Por último, en el tercer bloque, se compilaron todos los datos en un mapa digital colaborativo en el cual se pusieron marcadores, descripciones y fotografías sobre los puntos destacados en las salidas de campo.

Los resultados de esta investigación se han obtenido a partir de la categorización de las representaciones sociales de Reigota (1995): de tipo naturalista, antropocéntrica y globalizante. En las representaciones sociales de tipo naturalista, se suelen observar elementos como flora, fauna, montañas, lagos, ríos, etc. es decir, paisajes de diversos tipos, pero en los que predominan estos elementos propios del Medio Ambiente sin alteración 
humana. La categoría de las antropocéntricas muestra al ser humano como centro del entorno, para satisfacerle y donde existen servicios enfocados a las personas. La visión globalizante se caracteriza por un mayor grado de abstracción. Se establecen conexiones entre los servicios del medio urbano, su uso y sus conexiones: calles y recorridos, planos, etc. De esta manera el alumnado se hace una representación mental más aproximada a la realidad (Barraza, 2000) a partir de una comprensión compleja del entorno (Zabala, 1999), en este caso urbano, para abordar su transformación desde una perspectiva saludable y sostenible coherente con el ODS 11 de la Agenda 2030 (Naciones Unidas, 2015).

En otra categoría de análisis, se observaron dos tipos de enfoques, Jerez y Morales (2020): idealizados y problematizados. En los primeros se diferencian imágenes coloridas o descripciones connotativamente positivas, mientras que en los problematizados se resaltan aspectos a mejorar, imágenes oscuras y una visión en general crítica con el entorno.

Por último, se recogen las menciones de las emociones evocadas al pensar en su barrio. Estas emociones son las básicas de Ekman y Oster (1981) y otras que son susceptibles de ser sentidas al pensar en su entorno: alegría, tristeza, enfado, sorpresa,miedo, asco, culpabilidad, admiración, ninguna y otra.

\section{RESULTADOS Y DISCUSIÓN}

En los resultados obtenidos mediante la información recogida en pretest y postest, se han analizado las representaciones sociales según la categorización de Reigota (1995) que el alumnado tiene de su barrio, mediante descripciones gráficas y textuales; por otra parte, la visión que tienen de su entorno, bien idealizada o problematizada, categorización realizada en trabajos como el de Jerez y Morales (2020) y, por último, información sobre las emociones que experimentan apartir de las emociones básicas propuestas por Ekman y Oster (1981).

\subsection{Resultados globales}

Los resultados del grupo experimental se pueden observar en la tabla 1, con datos para el pretest y el postest, clasificados por cada categorización de representación social, enfoque y emoción.

Inicialmente, y en referencia a las representaciones sociales del pretest, se observa una predominancia de visión antropocéntrica del barrio, con 10 menciones en el grupo experimental. Además, este entorno es enfocado mediante una visión claramente idealizada, con 13 menciones. Las emociones más elegidas por el grupo experimental en el pretest fueron las de tranquilidad y ninguna, con 6 y 4 casos. Estas concuerdan con una percepción socioemocional positiva del barrio (Hernández y Caurín, 2020). 
Posteriormente, en el postest, la visión del alumnado en cuanto a las representaciones sociales cambia mayoritariamente hacia una globalizante (10 menciones). El enfoque problematizado también aumenta, pasando de 5 en el pretest, a 9 en el postest. En cuanto a las emociones, continúa predominando la tranquilidad, con 5 elecciones, y la alegría, con 6 .

\begin{tabular}{|c|c|c|c|c|c|c|c|c|c|c|}
\hline \multicolumn{6}{|c|}{ Representaciones sociales según la clasificación de REIGOTA } & \multicolumn{4}{|c|}{ ENFOQUE } & \multirow{3}{*}{$\begin{array}{c}\text { EMOCIONES a } \\
\text { partir de EKMAN } \\
\text { PRETEST / POSTEST }\end{array}$} \\
\hline \multicolumn{2}{|c|}{ NATURALISTA } & \multicolumn{2}{|c|}{ ANTROPOCÉNTRICA } & \multicolumn{2}{|c|}{ GLOBALIZANTE } & \multicolumn{2}{|c|}{ Idealizado } & \multicolumn{2}{|c|}{ Problematizado } & \\
\hline PRETEST & POSTEST & PRETEST & POSTEST & PRETEST & POSTEST & PRETEST & POSTEST & PRETEST & POSTEST & \\
\hline \multirow[t]{2}{*}{1} & & 2 & 4 & & 2 & 3 & 4 & & 2 & Alegría (3) / (6) \\
\hline & & & & & 1 & & & & 1 & Tristeza (0)/(1) \\
\hline \multirow[t]{9}{*}{2} & 1 & 4 & & & 4 & 6 & 2 & & 3 & Tranquilidad (6) / (5) \\
\hline & & & & 1 & 1 & & & 1 & 1 & Enfado (1) / (1) \\
\hline & & & 1 & 1 & 1 & & 1 & 1 & 1 & Sorpresa (1)/(2) \\
\hline & & & 1 & & & & 1 & & & Admiración (0) / (1) \\
\hline & & 1 & & 1 & & 1 & & 1 & & Miedo (2) / (0) \\
\hline & & & & 1 & & & & 1 & & $\operatorname{Asco}(1) /(0)$ \\
\hline & & & & & & & & & & Culpabilidad \\
\hline & & 3 & 1 & 1 & & 3 & 1 & 1 & & Ninguna (4) / (1) \\
\hline & & & & & 1 & & & & 1 & Otras $(0) /(1)$ \\
\hline
\end{tabular}

TABLA 1. Análisis comparativo pretest-postest del grupo experimental. Fuente: elaboración propia a partir de Reigota (1995) y Ekman y Oster (1981)

En la tabla 2 se exponen los datos recogidos del grupo control. En ellos, se observa una predominancia de la visión antropocéntrica del medio urbano, tanto en el pretest ( 9 casos) como en el postest (11 casos). La parte naturalista desaparece en el postest y no se observan cambios significativos entre las emociones evocadas en ambas fichas. La mayoría de ellas son emociones positivas, sobre todo referenciando la tranquilidad y la alegría.

A continuación, se ejemplificarán cada una de las categorías anteriores con un dibujo y descripción perteneciente a la muestra a quienes se les asignó previamente un código alfanumérico.

En la figura 1 se observa, específicamente, vegetación como una palmera, árboles de clara predominancia en el barrio. Cabe destacar que, al tratarse de la representación de una ciudad, estos elementos suelen ir acompañados generalmente de edificios, carreteras, coches, etc. en este caso, aparece una calzada, un banco y una estatua. Aun así, al haber elementos naturales, estamos ante una imagen que se clasificaría como naturalista. 


\begin{tabular}{|c|c|c|c|c|c|c|c|c|c|c|}
\hline \multicolumn{6}{|c|}{ Representaciones sociales según la clasificación de REIGOTA } & \multicolumn{4}{|c|}{ ENFOQUE } & \multirow{3}{*}{$\begin{array}{c}\text { EMOCIONES a } \\
\text { partir de EKMAN } \\
\text { PRETEST/POSTEST }\end{array}$} \\
\hline \multicolumn{2}{|c|}{ NATURALISTA } & \multicolumn{2}{|c|}{ ANTROPOCÉNTRICA } & \multicolumn{2}{|c|}{ GLOBALIZANTE } & \multicolumn{2}{|c|}{ Idealizado } & \multicolumn{2}{|c|}{ Problematizado } & \\
\hline PRETEST & POSTEST & PRETEST & POSTEST & PRETEST & POSTEST & PRETEST & POSTEST & PRETEST & POSTEST & \\
\hline \multirow[t]{2}{*}{1} & & 7 & 7 & 2 & 2 & 8 & 7 & 2 & 2 & Alegría (10) / (9) \\
\hline & & & & & 2 & & & & 2 & Tristeza $(0) /(2)$ \\
\hline \multirow[t]{9}{*}{2} & & 2 & 4 & & & 4 & 4 & & & Tranquilidad (4) / (4) \\
\hline & & & & 1 & & & & 1 & & Enfado (1) / (0) \\
\hline & & & & & & & & & & Sorpresa \\
\hline & & & & & 1 & & & & 1 & Admiración (0) /(1) \\
\hline & & & & & & & & & & Miedo \\
\hline & & & & & & & & & & Asco \\
\hline & & & & & & & & & & Culpabilidad \\
\hline & & & & 1 & & & & 1 & & Ninguna $(1) /(0)$ \\
\hline & & & & & & & & & & Otras \\
\hline
\end{tabular}

TABLA 2. Análisis comparativo pretest-postest del grupo control. Fuente: elaboración propia a partir de Reigota (1995) y Ekman y Oster (1981)

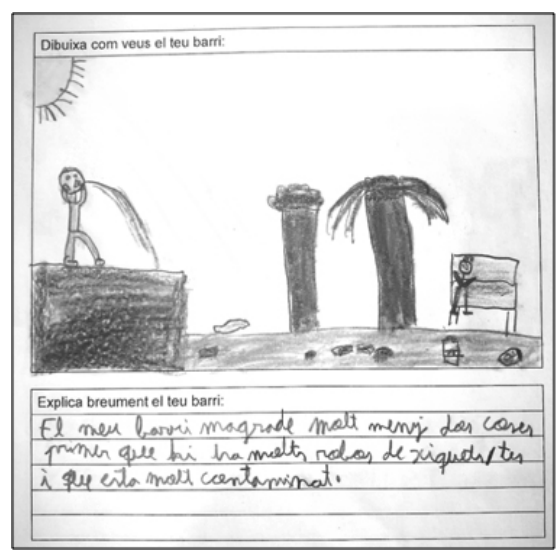

FiguRA 1. Representación social de la ciudad de tipo naturalista. Fuente: alumnado de la muestra (A7-E)

En la figura 2, se observan elementos del entorno urbano puramente centrados en el ser humano: edificios, coches, servicios como supermercados, la escuela, etc. Estos elementos son descriptivos de las representaciones sociales antropocéntricas, ya que están centradas en las personas. Estas no incluyen elementos naturales, sino aquellos que sirven a los humanos estrictamente hablando. 


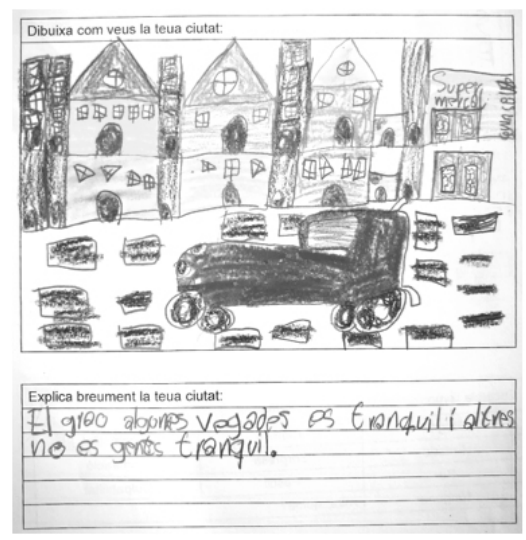

FIgURA 2. Representación social de la ciudad de tipo antropocéntrico. Fuente: alumnado de la muestra (A9-E)

En la figura 3, la visión del barrio es de tipo global, es decir, la ciudad y la sociedad están interconectadas. Se manejan conceptos como sociedad, globalización, etc. La citada figura representaría un ejemplo de enfoque idealizado, sobre todo observable en la descripción, ya que describe cosas de su agrado y hace afirmaciones como «es divertida». En cambio, los enfoques críticos, ponen de manifiesto algún problema, tanto en el dibujo de la figura 1 en el que se observa basura en el suelo, como en las explicaciones de ambas en las que se mencionan el ruido o la contaminación. Parece que el alumnado tiene presente el concepto de justicia ambiental, al plasmar esos problemas socioambientales en sus representaciones gráficas (Murga-Menoyo, 2018).

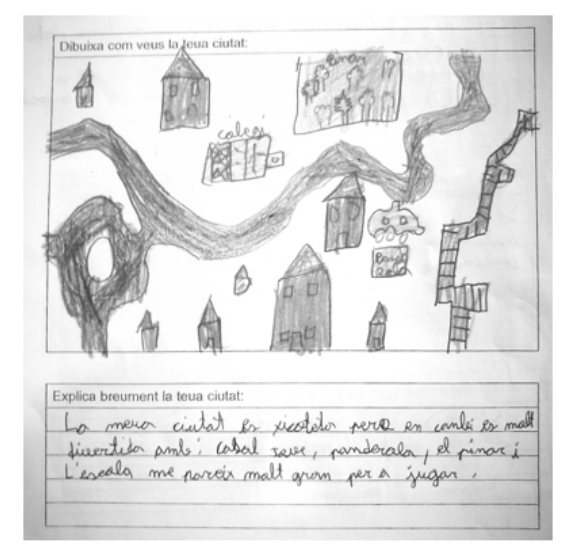

FIGURA 3. Representación social de la ciudad de tipo globalizante. Fuente: alumnado de la muestra (A13-C) 


\subsection{Relación entre representaciones sociales y emociones}

Una vez presentados los datos recogidos, el siguiente paso de análisis es el relacionar las variables mostradas anteriormente en la tabla 1, referente al grupo experimental. En este caso se observan ciertas diferencias entre pretest (figura 4) y postest (figura 5) representadas a continuación en forma de gráficas.

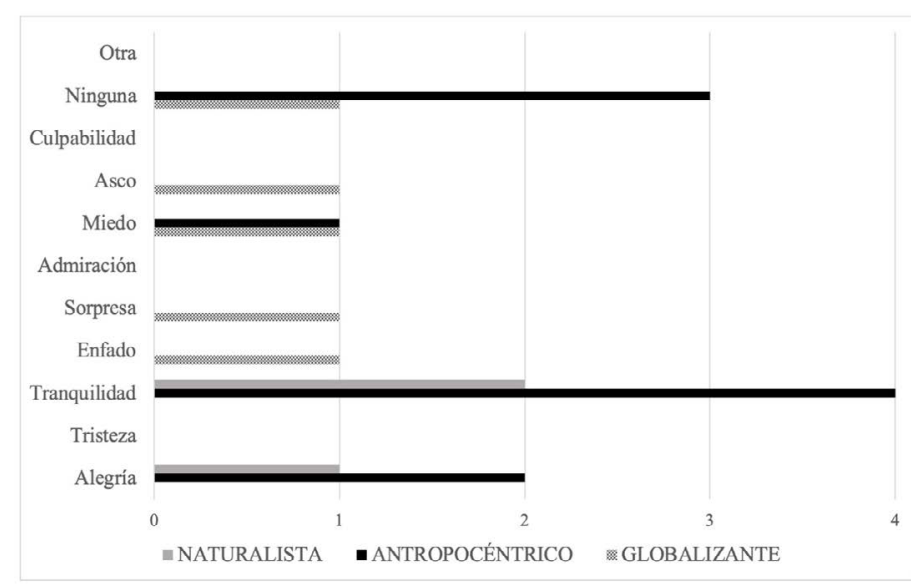

FiguRA 4. Gráfica que muestra la relación entre las representaciones sociales de Reigota (1995) y las emociones de Ekman y Oster(1981) en el pretest del grupo experimental. Fuente: elaboración propia

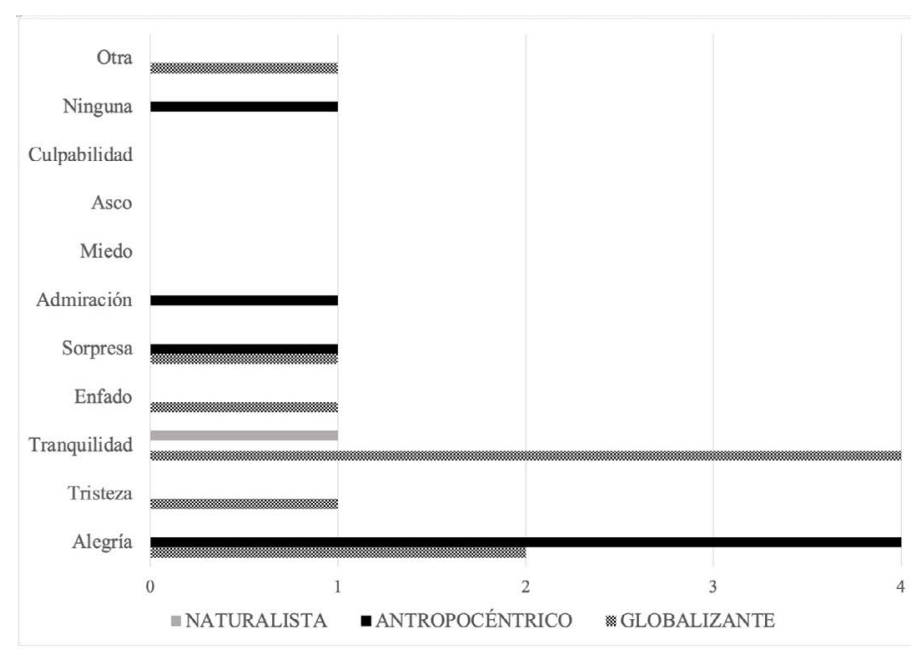

Figura 5. Gráfica que muestra la relación entre las representaciones sociales de Reigota (1995) y las emociones de Ekman y Oster (1981) en el postest del grupo experimental. Fuente: elaboración propia 
Respecto al grupo experimental en la figura 4 se evidencia una visión antropocéntrica del medio que gira hacia una visión globalizante después de la intervención (figura 5). El grupo control y experimental parten de unas representaciones sociales similares (figuras 4 y 6), pero, mientras que el cambio es pequeño en el grupo control (figura 7), en el experimental se ha evidenciado todo lo contrario (figura 5). Otro aspecto observable es que se han reducido también las menciones naturalistas existentes en un inicio. Parece ser que, la intervención en el aula y las vivencias del alumnado a través de las salidas de campo, mediante proyectos innovadores, contribuyen a cambiar las representaciones sociales de los escolares desde el espacio vivido (Souto, 2018).

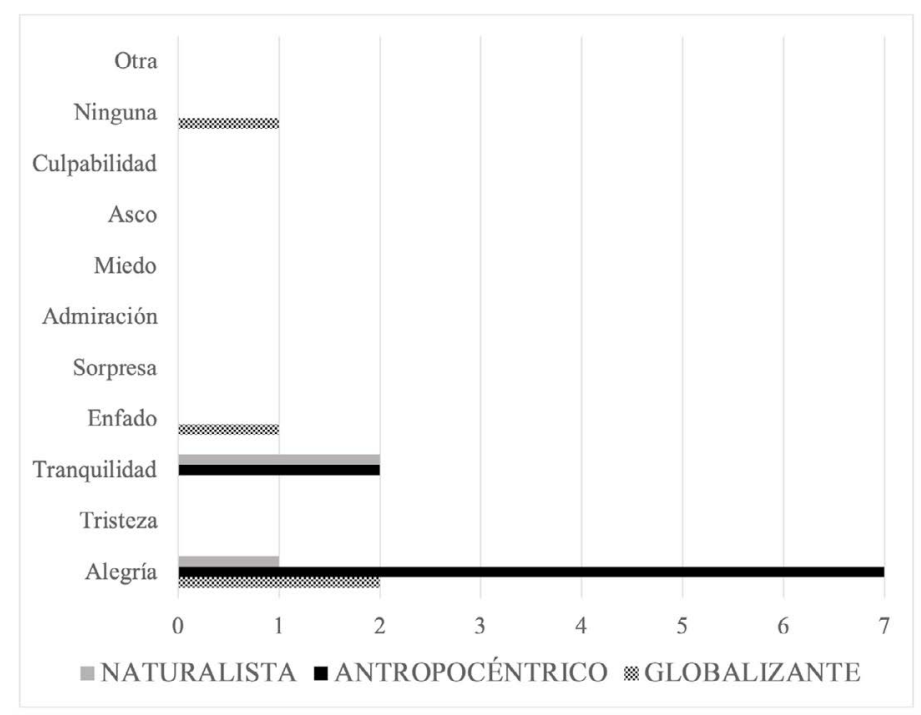

FIgURA 6. Gráfica que muestra la relación entre las representaciones sociales de Reigota (1995) y las emociones de Ekman y Oster (1981) en el pretest del grupo control. Fuente: elaboración propia

En cuanto a las emociones, destacan las de tranquilidad y alegría, aunque en un primer momento hay una diversificación evidente hacia emociones de todo tipo, sobre todo en el grupo experimental. En cuanto al grupo control, no existe tal diversificación, focalizándose, sobre todo, en emociones como alegría, tristeza o tranquilidad. Por tanto, se puede observar que, en general, las emociones positivas prevalecen aun cuando lo que cambian son las representaciones sociales. Pero en este caso las emociones de tranquilidad o alegría, en el contexto de los enfoques globalizantes, van asociadas a un conocimiento de las problemáticas, manifestando de esta manera el desarrollo de la inteligencia emocional (Goleman, 1995) y gestionando con ello las emociones 
desde la posibilidad de transformación del entorno urbano percibido, por otro más sostenible y saludable para vivir y ser vivido. Bisquerra (2003) nos corrobora que la educación emocional da respuesta a un conjunto de necesidades sociales que no quedan suficientemente atendidas en la educación más clásica y debe ser atendida en contextos más innovadores como las salidas al campo.

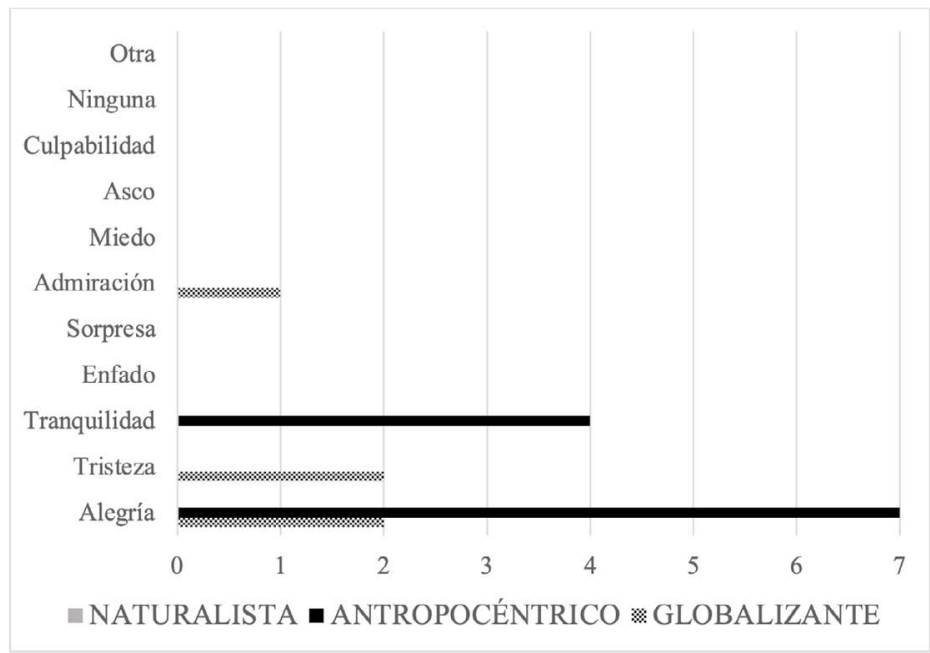

FIGURA 7. Gráfica que muestra la relación entre las representaciones sociales de Reigota (1995) y las emociones de Ekman y Oster (1981) en el postest del grupo control. Fuente: elaboración propia

\subsection{Relación entre enfoques idealizados o problematizados y emociones}

Atendiendo a los enfoques idealizados y problematizados se analiza si el alumnado de un grupo u otro han sabido pensar el medio de manera crítica o por el contrario no han detectado ningún problema.

En cuanto al grupo control, predomina la visión idealizada del medio urbano, tanto en pretest como en postest. Esta visión se ve conectada con las emociones positivas de alegría y tranquilidad mayoritariamente. Existe un ligero movimiento de 2 alumnos/ as hacia visiones problematizadas en el postest mediante anotaciones del tipo: «está contaminada, no me gusta que están reformando la ciudad...» (A7-C). Sánchez (2016), ya apuntaba esta relación entre emociones positivas e idealización del medio. Es decir, que no se observan cambios de enfoques evidentes entre las dos pruebas cuando nos 
referimos al grupo control. Sin embargo, en el grupo experimental sí se observan movimientos en este aspecto.

A continuación, en las figuras 8 y 9, se exponen en forma gráfica estos resultados:

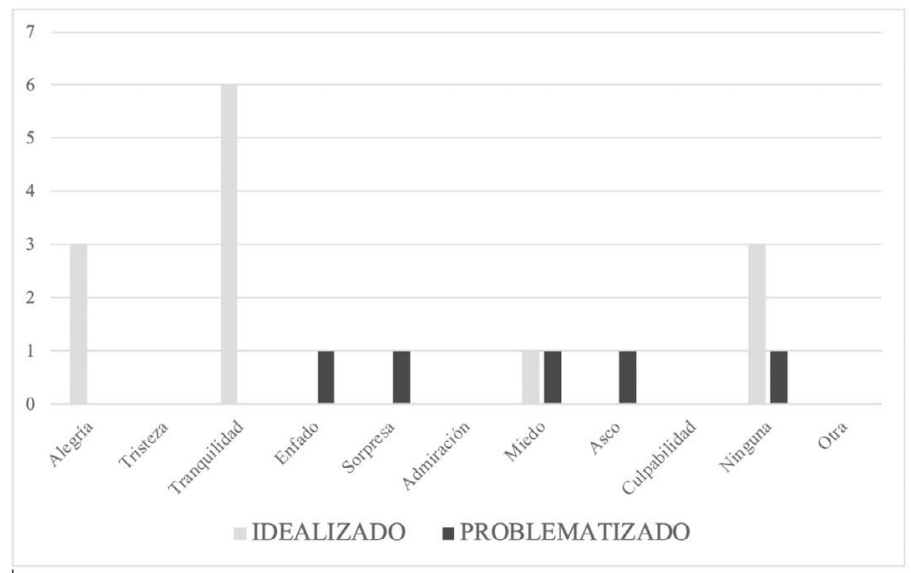

FiguRA 8. Gráfica que muestra la relación entre los enfoques y las emociones de Ekman y Oster (1981) en el pretest del grupo experimental. Fuente: elaboración propia

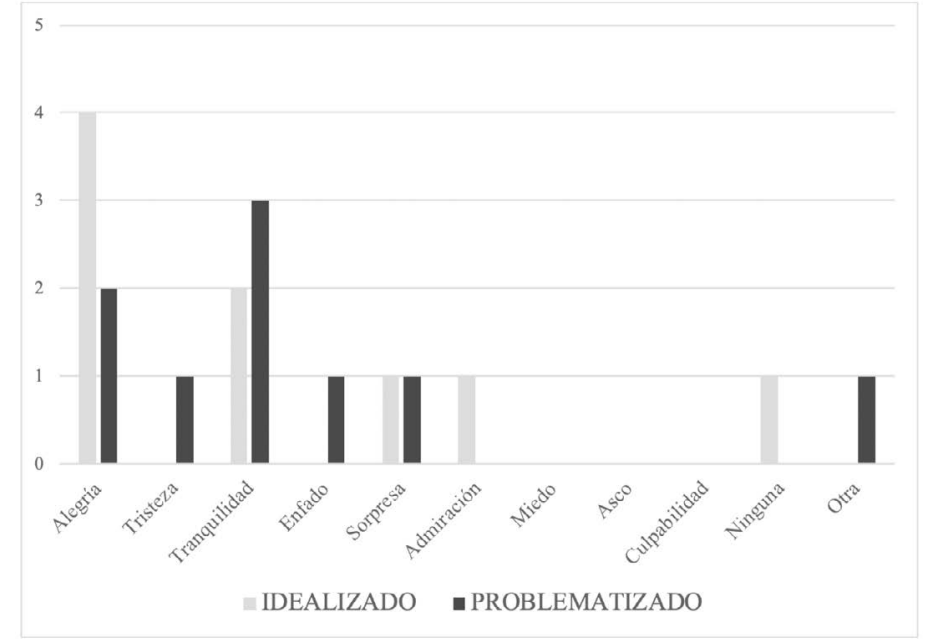

Figura 9. Gráfica que muestra la relación entre los enfoques y las emociones de Ekman y Oster (1981) en el postest del grupo experimental. Fuente: elaboración propia 
En la figura 8 e parte de un enfoque idealizado del barrio con 13 alumnos/as (de 18 miembros de la muestra), con predominancia de las emociones de alegría, tranquilidad o ninguna, resultados muy similares al del grupo control. En el postest, en cambio, aumenta la visión problematizada con 9 representaciones (figura 9). Algunas citas referentes a esta visión crítica serían: «a mi me gusta mucho, pero hay muchas obras» (A6-E) o «es pequeña, hay muchos habitantes y está muy contaminada» (A4-E). En cuanto a las emociones evocadas parece que se mantienen mayoritariamente positivas, lo cual remite a que el alumnado detecta más problemáticas después de la intervención, pero continúa sintiendo el barrio de manera positiva y abierto a la mejora. Este hecho puede estar relacionado con el concepto de transformar las emociones en acciones positivas, la llamada ecología emocional (Soler y Conangla, 2004).

\subsection{Comparativa de resultados entre grupo control y grupo experimental}

Para finalizar este apartado de análisis y discusión de resultados, se procedió a comparar los datos del grupo control y experimental para extraer las posteriores conclusiones. En las siguientes figuras 10 y 11 se observan estas comparativas.

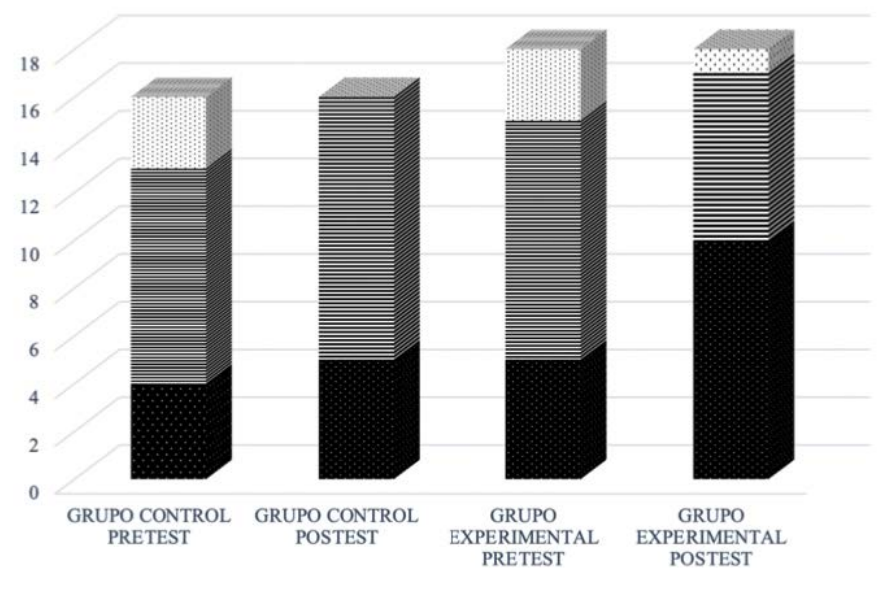

- GLOBALIZANTE $\equiv$ ANTROPOCÉNTRICO NATURALISTA

FiguRA 10. Análisis comparativo entre las representaciones sociales de Reigota (1995) de los grupos control y experimental. Fuente: elaboración propia 


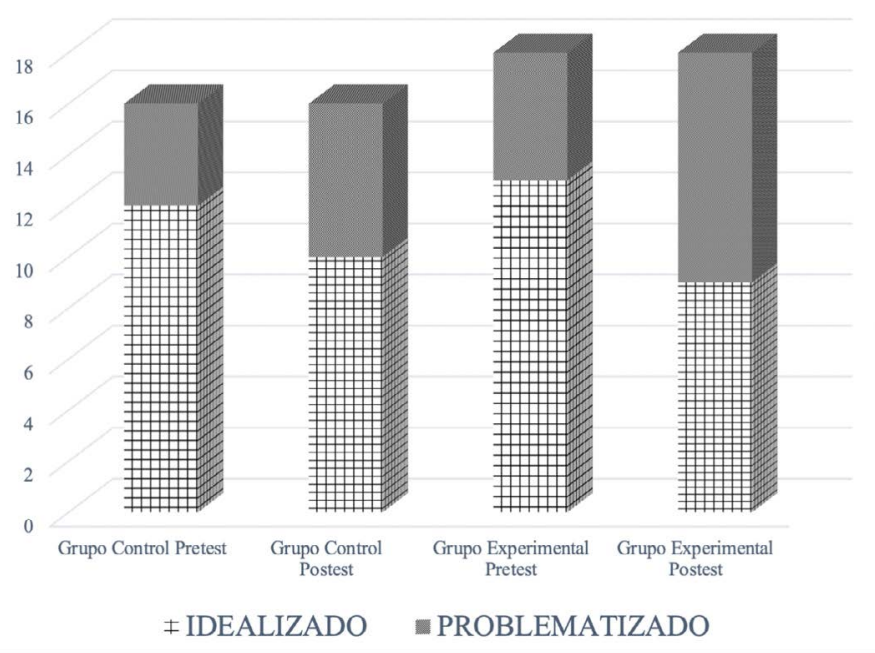

FIGURA 11. Análisis comparativo de los enfoques idealizados y problematizados de los grupos control y experimental. Fuente: elaboración propia

En la figura 10 se puede observar esa predominancia antropocentrista del grupo control en ambas pruebas, mientras que en el grupo experimental parte de una visión parecida a la del grupo control, en el postest, la representación globalizante cobra protagonismo y marca la diferencia. Parece que hay indicios en los que, tras una intervención educativa, las representaciones sociales varían.

En este punto destacamos también la relación entre emociones y actitudes, ya que tanto las emociones positivas como las negativas nos predisponen a la acción, pues el enfado o la tristeza pueden desembocar en acciones resolutorias y la alegría y la tranquilidad en acciones conservadoras. Aunque es antigua, la mejor definición de actitudes es la de Rodríquez y Seoane (1989) que aclara las consideraciones que acabamos de hacer. Se define la actitud como una organización persistente en el tiempo, de creencias y conocimientos que están basados en una carga afectiva (emocional) que predispone a favor o en contra de objetos, situaciones o personas; esto desemboca en una acción coherente y conecta percepciones, emociones y acciones. Souto (2018) nos habla de la percepción de los niños sobre el espacio y de la relación entre el pensar y el sentir que surge de las interacciones personales y colectivas que estructuran los elementos del espacio escolar y por tanto el espacio vivido.

Como se ha mencionado antes, estas evidencias observables van siempre de la mano de emociones connotativamente positivas, es decir, el alumnado de la muestra siente el barrio con alegría y tranquilidad, independientemente de cómo lo vea o el enfoque que le den. 
En la figura 11 se observa un cambio hacia los enfoques problematizados: en el grupo control de manera más sutil (de 4 estudiantes en el pretest a 5 en el postest), y en el grupo experimental de manera más representativa en este sentido (de 5 a 9 estudiantes). Es decir, se observa una visión idealizada en su mayoría, sobre todo en el grupo control, donde esa mayoría se mantiene en ambas pruebas, mientras que en el grupo experimental los enfoques se igualan en el postest y el crítico gana protagonismo.

A partir de todo esto se evidencia que las salidas de campo son un recurso valioso para problematizar el entorno urbano y participar de la ciudadanía crítica, tal y como manifestaban (Santana Morales y Souto, 2019) en el ámbito de los parques naturales. Esto coincide con la investigación de Berger et al. (2009) que dan a la escuela el papel de crear medios que capaciten al alumnado con actitudes críticas que le permitan participar activamente en la construcción de la sociedad.

Frente a estas problemáticas, cada vez con mayor insistencia se le pide a la escuela que se haga cargo de ellas a través del proceso formativo. Precisamente entre los objetivos centrales de la educación escolar destaca el proporcionar a los estudiantes una formación integral, que les ayude a estructurar su identidad y a desarrollar sus capacidades para participar efectivamente en la construcción de la sociedad, es decir, se pone de manifiesto la necesaria participación de la infancia en las decisiones sobre las ciudades que habitan (Tonucci, 2015).

\section{CONCLUSIONES}

A modo de conclusiones para proseguir cabe destacar, en primer lugar, los siguientes aspectos sobre las representaciones sociales que el alumnado tiene sobre su barrio y su conexión con las emociones:

- El análisis de las representaciones sociales del entorno del alumnado de la muestra verifica que en el pretest hay menor diversidad de emociones que en el postest y esto se ve de forma más evidente en el grupo experimental que en el control debido a las salidas de campo.

- Las emociones connotativamente positivas, como la alegría y la tranquilidad, están asociadas, en su mayoría, a enfoques idealizados, mientras que las negativas como el enfado o la tristeza se han relacionado con los enfoques problematizados. Esto se observa principalmente en el pretest de todo el alumnado de la muestra.

- Se observan emociones positivas vinculadas a enfoques problematizados en el postest del grupo experimental al transformar esas emociones en acciones positivas de cambio y de participación ciudadana en su barrio.

- La visión que el alumnado de la muestra tiene sobre su entorno próximo parte, mayoritariamente de una concepción antropocéntrica del barrio. Ésta se mantiene en el 
grupo control, pero se modifica en el experimental hacia una visión globalizante y más biocéntrica después de la intervención.

- Los enfoques que el alumnado realiza de su barrio y ciudad parten de una visión idealizada del medio. Este enfoque se mantiene en el grupo control, pero evoluciona en el experimental, identificando un enfoque problematizado en la mitad de la muestra, debido a las salidas de campo.

Así mismo se deben subrayar las siguientes consideraciones referentes a la intervención en el aula, las salidas de campo y las problemáticas detectadas:

- La intervención en el aula a través de las salidas de campo, es capaz de modificar las representaciones sociales del alumnado de la muestra.

- La escuela es un agente dinamizador para fomentar el pensamiento crítico, al aumentar la visión problematizadora del medio después de las salidas de campo y el trabajo en el aula.

- Los proyectos innovadores de intervención-acción en aula relacionados con las salidas escolares, desde la metodología científica propia del trabajo de campo, permite aprender la realidad desde el espacio vivido, superando con ello los entornos urbanos percibidos, reflejadas en las ciudades tipo genéricas de los manuales escolares.

Por todo ello se corrobora que la escuela puede ayudar al cumplimento del ODS 11, utilizando como recurso didáctico la salida de campo, contribuyendo a la conceptualización de la ciudad desde el espacio emocionalmente vivido por el alumnado, al evidenciar la problematización del entorno urbano y la necesidad de poder mejorarlo a partir de las emociones de los propios escolares. Permitiendo con ello el ejercicio de una ciudadanía crítica por parte de la infancia, en el marco transformador orientado por la Agenda 2030, para construir desde el presente las ciudades sostenibles del futuro.

\section{REFERENCIAS BIBLIOGRÁFICAS}

Abarca, M., (2003). La Educación Emocional en la Educación Primaria: currículo y práctica. Tesis doctoral. Universitat de Barcelona.

Barraza, L. (2000). Educar para el futuro: En busca de un nuevo enfoque de investigación en Educación ambiental. En Memorias Foro Nacional de Educación Ambiental (pp. 253-260). UAA, SEP y SEMARNAP. 
Berger, C., Milicic, N., Alcalay, L., Torretti, A., Arab, M.P., Justiniano, B. (2009). Bienestar socio-emocional en contextos escolares: la percepción de estudiantes chilenos. Estudios sobre educacion, 17, 21-43.

Bisquerra, R. (2000). Educación emocional y bienestar. Barcelona: Praxis.

Bisquerra, R. (2003). Educación emocional y competencias básicas para la vida. Revista de Investigación Educativa, 1 (21), 7-43.

Bisquerra, R. (2005). La educación emocional en la formación del profesorado. Revista interuniversitaria de formación del profesorado, 19(3), 95-114.

Bisquerra R., y Pérez Escoda, N, (2007). Las competencias emocionales. Educación XXI, 10, 61-82. DOI: https://doi.org/10.5944/educxx1.1.10.297

Bisquerra, R. (2011). Educación emocional. Propuestas para educadores y familias. Bilbao: Desclée de Brower.

Boira, J., Reques, P., Souto, X. (1994). Espacio subjetivo y geografía. Valencia: Nau Llibres. Bourdieu.

Breda, T. V., y García de la Vega, A. (2018). El desarrollo del razonamiento geográfico a través de una propuesta ludo-didáctica en la ciudad. Didáctica Geográfica, (19), 197-220. DOI: https://doi.org/10.21138/DG.422

Capel, H. (1973). Percepción del medio y comportamiento geográfico. Revista de Geografía, 7, 58-150.

Elliott, J. (1993). El cambio educativo desde la investigación-acción, trad. P. Manzano,

Ekman, P., y Oster, H. (1981). Expresiones faciales de la emoción. Estudios de psicología, 2(7), 115-144.

Fernández Berrocal, P. y Extremera, N. 2009. La Inteligencia Emocional y el estudio de la felicidad. Revista Interuniversitaria de Formación del Profesorado, $66(23,3)$, 73-8.

González, A. (2019). Percepción crítica y emocional del entorno urbano en la infancia: estudio de casos a partir de las salidas de campo y el mapeo digital. Trabajo Final de Máster inédito. Valencia: Universitat de València.

Goleman, D. (1995). Emotional Intelligence. New York: Bantam Books. (Trad. Cast., Kairós, 1996).

Gupta, A., Love, A., Kilpatrick, L. A., Labus, J. S., Bhatt, R., Chang, L., Tillisch, K., Naliboff, B. \& Mayer, E. A. (2018). Morphological Brain Measures of CorticoLimbic Inhibition Related to Resilience. Journal of Neuroscience Research. 95(9), 1760-1775. DOI: https://doi.org/10.1002/jnr.24007.

Hannoun, H. (1977). El niño conquista el medio. Buenos aires: Kapelusz. 
Hernández, S. y Caurín, C. (2020). Gestión de conflictos en contextos educativos e integración familia-escuela. Madrid: INISEG. SOTEC

Jerez, M. (2018). El aprendizaje de la cartografía personal: estudio de casos en el primer ciclo de Educación primaria. Trabajo Final de Máster. Universitat de Valéncia. Inédito.

Jerez, M. y Morales, A. J. (2020). Cartografías personales y ciudadanía crítica: una investigación didáctica en educación primaria. Iber: Didáctica de las ciencias sociales, geografía e historia, (98), 8-13.

Jodelet, D. (1986). La representación social: fenómenos, concepto y teoría. Moscovici, Serge (comp.), Psicología Social II (pp. 469-494). Barcelona: Paidós,

Lefebvre, H. (2000). La production de l'espace. Paris: Anthropos.

López Cassà, È. (2005). La educación emocional en la educación infantil. Revista interuniversitaria de Formación del Profesorado, 19(3), 153-167.

López Facal, R. (1987). Estudiar la ciudad, transformar la ciudad. Cuadernos de Pedagogía, 153, 24-26.

Mora, F. (2018). Mitos y verdades del cerebro. Barcelona: Paidós

Morales, A. J., Santana, D. y Sánchez, L. (2017). Identidades territoriales y educación ambiental. Del paisaje emocional al paisaje cultural. Íber, Didáctica de las Ciencias Sociales, geografía e Historia, 89, 12-17.

Moscovici, S. (1981). El psicoanálisis, su imagen y su público. Buenos Aires: Huemal.

Muntañola, J. (1987). Els nens i la seva ciutat. Barcelona-New York. Colección Descobrir el medi urbá, 5. Barcelona: Institut d’Ecologia Urbana. (Edición bilingüe catalán/ castellano).

Murga-Menoyo, M. A., y Novo, M. (2017). Sostenibilidad, desarrollo «glocal»y ciudadanía planetaria. Referentes de una Pedagogía para el desarrollo sostenible. Teoría de la Educación. Revista Interuniversitaria, 29(1), 55-78. DOI: https://doi. org/10.14201/teoredu2915579

Murga-Menoyo, M.A. (2018). La Formación de la Ciudadanía en el Marco de la Agenda 2030 y la Justicia Ambiental. Revista Internacional de Educación para la Justicia Social, 7(1), 35-52. DOI: https://doi.org/10.15366/riejs2018.7.1.002

Naciones Unidas, (2015). Transformar nuestro mundo: la Agenda 2030 para el desarrollo sostenible. Disponible en: https:/unctad.org/meetings/es/SessionalDocuments/ ares70d1 es.pdf

Reigota, M. (1995). Meio ambiente e representação social. São Paulo: Cortez.

Rodríguez, M. (2005). Introducción a la educación socioambiental desde la pedagogía social. Málaga: Aljibe. 
Rodríguez, A. y Seoane, J. (1989). Creencias, actitudes y valores. Madrid: Alhambra Rubia, F. J. (2007). El cerebro nos engaña. Barcelona: Temas de hoy.

Salovey, P. y Mayer, J. (1990). Emotional Intelligence. Imagination, Cognition and Personality, 9, 185-211. DOI: https://doi.org/10.2190/DUGG-P24E-52WK-6CDG

Sánchez, L. (2016) Las representaciones sociales del concepto de medio ambiente y su incidencia emocional en el tercer ciclo de Educación Primaria. Estudio de casos. Trabajo Final de Máster. Universitat de València. Inédito.

Santana, D., Morales, A. J. y Souto, X. M. (2019). ¿Cómo favorecer la ciudadanía critica con la ayuda de la educación ambiental? En: M. Ferreras-Listán, O. MorenoFernández y M. ${ }^{a}$. Puig-Gutiérrez, (coords.) Innovación e investigación en la formación inicial del profesorado de Infantil y Primaria desde las didácticas de las Ciencias Experimentales y Sociales (pp. 77-100). Barcelona: Octaedro,

Soler, J., y Conangla, M. M. (2004). La ecología emocional. Barcelona: Amat.

Souto, X. M. (2017). Los métodos didácticos en la enseñanza del espacio geográfico. En: R. Sebastià y E. Mª .Tonda (coords.). Enseñanza y aprendizaje de la geografía para el siglo XXI (pp. 73-96). San Vicent del Raspeig (Alacant): Universitat d'Alacant,

Souto, X. (2018). La geografía escolar: deseos institucionales y vivencias de aula. Boletín de La Asociación de Geógrafos Españoles, 79, 2757, 1-31. DOI: https:// doi.org/10.21138/bage. 2757

Stake, R. (2005). Investigación con estudio de casos. Madrid: Ediciones Morata.

Taylor S. y Bogdan R. (1992) Introducción a los Métodos Cualitativos de Investigación. Barcelona, Buenos Aires y Mexico: Paidós Básica. Madrid: Morata.

Tatjer, M. (2001). Ensenyar la ciutat o aprendre de la ciutat: l'exemple de Barcelona, Temps d'educació, 26, 67-85.

Taylor S. y Bogdan R. (1992) Introducción a los Métodos Cualitativos de Investigación. Barcelona, Buenos Aires y Mexico: Paidós Básica. Madrid: Morata.

Tomé, S. (1994). Los problemas urbanos. Consideraciones teóricas. En I. Dopazo et al., El estudio geográfico de los problemas urbanos. Orientación y praxis didáctica (pp. 5-28).Valencia: Nau Llibres,

Tonucci, F. (2009). Ciudades a escala humana: la ciudad de los niños. Revista de Educación, extra, 1, 147-168.

Tonucci, F. (2015). La ciudad de los niños. Barcelona: Graó.

UNICEF (2016). Convención sobre los Derechos del Niño. Recuperado en https://www. un.org/es/events/childrenday/pdf/derechos.pdf

Zabala, A. (1999). Enfoque globalizador y pensamiento complejo: una respuesta para la comprensión e intervención en la realidad. Barcelona: Graó. 